\title{
A Retrospective Analysis of Owner-reported Reasons for Canine Illness and Injury Visits in Veterinary General Practice: A List of the Top 50\% of Complaints
}

Gilbert Patterson ( $\sim$ gilbert.patterson@lmunet.edu )

Lincoln Memorial University https://orcid.org/0000-0001-6155-0311

Michele 0. Trofatter

Lincoln Memorial University

Kathryn E. Daily-Trude

Lincoln Memorial University

Rebecca L. Pierce

Lincoln Memorial University

Stanley R. Robertson

Lincoln Memorial University

John J. Weale

Lincoln Memorial University

Jason W Johnson

Lincoln Memorial University

Research article

Keywords: Canine Health, Illness, Injury, General Veterinary Practice, Top 10 Complaints

Posted Date: April 1st, 2020

DOI: https://doi.org/10.21203/rs.3.rs-19713/v1

License: (c) (1) This work is licensed under a Creative Commons Attribution 4.0 International License.

Read Full License 


\section{Abstract}

Background Veterinarians are required to use critical thinking and communication skills to proficiently guide a client through available options for disease treatment or management. While multiple viable approaches exist for treating common problems, data describing the actual types of owner-reported complaints most often encountered in general veterinary practices is lacking. An understanding of the nature and distribution of common conditions, as reported by the owner, is a key step in providing the evidence-based foundation directing further efforts toward solutions to overcome barriers in general practice, veterinary-care delivery. Methods A retrospective analysis of common canine owner-reported complaints presented in general veterinary practice over a one-year period was performed. Data was collected from participating practices, cleaned, and analyzed to reflect the top $50 \%$ new presenting complaints of owners at all participating practices for canine patients with an illness or injury during their visit. Results The outcome is a comprehensive list ranking the top canine owner-reported presenting illnesses/injuries complaints seen by general veterinary practices included in the study. Conclusions These results provide evidence-based knowledge of the distribution of owner complaints potentially encountered in general practice, providing justification for the need to emphasize certain clinical case presentations in veterinary educational curriculums. Being prepared with such knowledge, veterinary students can be empowered with the necessary skills and tools required to provide informed, ethical, and affordable choices for canine care in the general veterinary practice setting. This information also provides the scientific foundation for canine conditions which may benefit from additional evidencebased trials to better identify the outcomes of spectrum of care interventions for benefits to the owner, the canine, and the veterinarian.

\section{Background:}

Veterinarians strive to prevent and relieve animal suffering and promote animal health and welfare. During their education and clinical training in veterinary school, they are introduced to the newest techniques and technology currently available in order to provide a "gold standard" for patient care. ${ }^{1,2,3}$ In an ideal world, these various options would be available to them, their clients and their patients at all times in the course of routine clinical care. However, upon entering practice, veterinarians soon realize that meeting the needs of their patients and clients requires making safe, effective, and economically viable decisions, which may not always be in line with the preeminent technique or treatment available and learned in veterinary school. ${ }^{2,3}$

Such decisions are affected by a number of variables, including client finances, owner perceptions and attitudes toward the animal, and the nature and severity of the illness. ${ }^{4,5}$ Nevertheless, the course of action recommended by the clinician must be scientifically verifiable, ethically sound, and fairly priced in order for the veterinarian to continue practicing high quality medicine and maintain economic productivity. ${ }^{4,5,6,7}$ Given the wide spectrum of diagnostic and treatment options available to veterinarians, it is important that veterinarians are adequately trained to recognize situations where their ability to be 
flexible and innovative in their approach to treatment may be required. While every encounter with a patient and client is different, the veterinarian's ability to recognize that there may be multiple treatment approaches to a common problem is paramount to upholding modern standards of care. ${ }^{1,2,3} \mathrm{An}$ understanding of what these common problems are, and the frequency with which they might be encountered, is integral to preparing clinically competent veterinarians. ${ }^{1,3}$ From this information, the training of future veterinarians to be receptive to alternative approaches to treatment and care can be better directed toward areas where it will be most effective. By understanding the types of illnesses and or injuries that will most frequently prompt a pet owner to seek veterinary care, veterinary curriculums can be better tailored to prepare future veterinarians for situations they will encounter in daily general practice ${ }^{1,4}$

In this study, the frequency with which specific types of presenting canine complaints, as reported by the owner, are encountered in general veterinary practice is described and analyzed. To accomplish this, a retrospective analysis of owner identified complaints for common canine conditions that presented at general veterinary practices, over the course of a one-year period was conducted. Data was gathered from participating clinics and analyzed to reflect the top $50 \%$ presenting injury or illness complaints. The purpose of this information is to provide a better understanding of the types of illnesses and or injuries that will most frequently prompt a pet owner to seek veterinary care, so that modern veterinary curriculums can be better tailored to prepare future veterinarians for situations they will encounter in daily general practice. Additionally, this information serves to provide the broader veterinary profession and community with evidence-based data supporting the ongoing need to maintain innovative, low-cost, and ethical treatment solutions in order to maximize the accessibility and availability of veterinary care.

\section{Results:}

After the inclusion criteria was set and the data was cleaned through, the $65,000+$ data points started with were classified to 21,453 owner-reported complaints which met the inclusion criteria. These 21,453 complaints represented 14,693 canine visits, with 5,376 of the visits including more than one ownerreported complaint. There were ten (10) complaints accounting for $49.7 \%$ of all complaints captured, which for ease of reporting purposes, has been rounded up to describe the top $50 \%$ of complaints encountered in general veterinary practice. The top ten (10) owner reported complaints were, in descending order of frequency: ear issues (1471), vomiting (1459), skin issues (1448), mass (1122), diarrhea (1045), not eating (954), eye issues (887), lameness (877), lethargic (763), and coughing (633). See Fig. 1.

Within the top $50 \%$ of owner-reported complaints,there was found to be a steady distribution and decline between the complaints. There was no sudden drop in the numbers associated with the top $50 \%$ of owner-reported complaints suggesting any complaint within that data set was overwhelmingly making up a majority of the top $50 \%$ of owner-reported complaints seen within the study. See Fig. 2.

Data was stratified according to sex, $\mathrm{n}=21,453$ and $39 \%(4,100)$ were female spayed, $10 \%(1,013)$ were female, $32 \%(3,493)$ were male neutered, $13 \%(1,430)$ were male, and $6 \%(623)$ were unknown or not 
reported. See Fig. 3. Data for age was available for 11,659 canine visits; the remaining 3,033 did not have an age recorded. The minimum age was one month or less (0.08 years) and the maximum age was 28 years. Average age was 6.8 years. Due to the lack of standardization of breed names between clinics and electronic data systems, a representation of the breeds could not be reliably captured.

An analysis of the owner-reported complaint data according to sex revealed no significant differences ( $p$ $>0.05$ ) for the top 50\% complaints. See Table 1 and Fig. 4.

\section{Discussion/conclusions:}

Similar studies describing the prevalence of specific conditions encountered in general veterinary practice have been conducted and published internationally, particularly in the UK. ${ }^{8,9,10}$ Of these, the most comprehensive study of general veterinary practice reported otitis externa, periodontal disease, and anal sac impaction, as the most frequently cited disorders. ${ }^{8}$ In the US, the most extensive database describing common canine conditions is maintained by Banfield Pet Hospital, which publishes regular State of Pet Health reports, based on data gathered from its nearly 1000 veterinary hospitals across the US and Puerto Rico. Within the most recent Banfield State of Pet Health report, the top five most commonly diagnosed canine conditions are dental calculus, overweight, otitis externa, patellar luxation, and nuclear sclerosis. ${ }^{11}$

Additionally, pet insurance providers have also published canine claim information to identify top conditions for over the last the past fifteen years. ${ }^{8,9,10}$ In the most recent claims reported by the insurance provider Nationwide, the top ten conditions were (in order of frequency), skin allergies, ear infection, mass, skin infections, diarrhea, vomiting, arthritis, dental disease, bladder infections / urinary tract infections, and anal gland inflammation or infection. ${ }^{12,13}$

While the results of these various studies and databases do not differ dramatically from those of this study, there are some fundamental differences in the source material of these data sets, and the approach taken in this study, which are important to consider. Most notably, all of these studies and reports categorized conditions as they have been diagnosed by a veterinarian, whereas in this study the focus was on owner-initiated complaints of illness or injury. While the difference in this approach may not seem substantial, they do in fact reveal a number of noteworthy features that become critically important when determining how to best apply this information.

By comparing the results of this study to others that focus on veterinary diagnoses, the knowledge gap between the average pet owner and veterinarian is revealed. This explains the more generalized, ownerinitiated "skin" condition described in this study's data, compared to the "skin allergies," and "skin infections" that were captured in the insurance data following a formal diagnosis from a veterinarian. For most cases of canine injury or illness, veterinarians generally rely on the owner to recognize that a 
condition has become problematic enough to warrant a veterinarian's attention. Once the animal is brought to the clinic, the veterinarian will work with whatever information is provided by the owner in order to help determine the diagnosis. As supported by this study, often this information is limited to a generalized body part, behavior, or that the animal just doesn't seem "right." Working with this incomplete picture, there could be any number of potential causes to the reported problem, but it is the veterinarian's job to determine the correct etiology and proceed with the appropriate treatment. It is within this gap in knowledge that the veterinary practitioner operates, utilizing their medical knowledge and experience to bridge the divide between complaint and a final diagnosis. The results of this study provide justification for veterinary curriculums to train students to recognize common clinical presentations as they are reported in practice, and to be adequately prepared to address the variety of distinct and overlapping etiologies that cause them.

Additionally, the results of this study reveal a discrepancy in how pet owners and veterinarians prioritize the reporting of specific conditions. It is worth noting that frequently occurring clinical signs in this study, such as not eating, lethargy, or coughing do not show up in the insurance data, or the diagnostic surveys compiled in the UK and Banfield. ${ }^{8,11}$ Conversely, conditions such as obesity and periodontal disease are commonly diagnosed by veterinarians, but according to the results of this study, are less likely reasons for a pet owner to seek veterinary care, with the owner-reported symptoms "Mouth" and "Overweight" making the list at spots 19 and 158 respectively. Both symptoms well under the top $50 \%$ of reasons an owner would take their canine to the vet and with no guarantee that an owner seeking help for a mouth problem in their canine would translate to periodontal disease. This difference again helps to illustrate the value of a veterinarian's diagnostic eye to a pet's overall health. While conditions such as obesity and dental disease may not be bringing many pets in the clinic's doors, this study provides evidence of the need for further client education to recognize the importance of treating chronic conditions in their pets.

It should be stated further that data from insurance claims cannot accurately reflect the overall canine population, as it is biased by the presupposition of the pet's insurance status. In the US, according to the North American Pet Health Insurance Association (NAPHI) just over $1 \%$ of dog owners have pet insurance for their animals. While the pet health insurance industry is rapidly growing within the US, this number is hardly representative of the general population of dog owners. ${ }^{14}$ The data from this study, collected from independent general veterinary practices across several states, while not a representative sample, are a better representation of canine illness and injury presentations based on owner-reported complaints than has been previously available from other sources. The results presented are no longer the empirical representations based on years of experience, or the claims data from a small, insured population, but are data-driven and based on standardized collection of information from the general canine ownership population.

Evidence-based data, such as the list of common complaints derived in this research, is important in the future of veterinary medicine, as well as in the education of future veterinarians. ${ }^{1,2,3}$ Knowing the full spectrum of care diagnostic techniques and treatment modalities does not guarantee access, availability, or affordability. Confounding factors such as owner perceptions, attitudes, and the financial situation of 
the owner impact treatment outcomes and decisions which are made by the owner instead of the veterinarian. Continuing to provide information for owners, based on scientific investigation and research can support informed care decisions, increase shared decision making, increase owner compliance and satisfaction with suggested care modalities, and support access to care with a range of valid proven treatments. Education of future veterinarians is also positively impacted by information derived from critical, evidence-based research as a foundation for students' critical thinking skills, communication training, and increased knowledge base.

\section{Methods:}

Thirteen veterinary practices agreed to participate in the data acquisition process for this study. These practices are part of a broader national clinical affiliate network providing experiential learning clinical rotations for a North American veterinary school that utilizes a hybrid distributive model as part of a fourth-year veterinary student curriculum. Clinical affiliates were asked directly for their voluntary participation in the study, and those that agreed were part of the convenience sample pool to be studied. The participating practices were categorized by internal clinical affiliate criteria as predominately or exclusively small animal general veterinary practices and represented six US States. Three of the practices used paper medical records or a combination of paper medical records and electronic billing. The remainder of practices relied entirely on electronic systems for medical records and billing, with a total of five different systems represented. Five of the captured data sets were deemed unusable for analysis either because of incomplete data received from the site or incompatible format used by the site. These were not included in the final analysis.

Data was gathered over a one-year period, counting back 365 days from the date of data capture. Inclusion criteria from the collected canine data was restricted to specific, owner-initiated complaints for canine illness or injury. In order to focus exclusively on visits that were prompted by the pet owner, wellness visits, vaccinations, repeat or recheck visits for the same illness or injury, non-owner initiated (groomer, boarding, etc.), diagnostic visits, surgical/pre-surgical visits, and therapeutic visits (acupuncture, chiropractic e.g.), quality of life assessments, planned euthanasia, or chemotherapy visits were excluded. Data capture also included the canine patient's age, sex, and breed.

Over 65,000 visits or qualifying data points were examined for inclusion/exclusion criteria. Up to three complaints per visit were captured for analysis, although occasionally more were listed. Due to differences among the various medical record systems as well as variation among individual clinic staff capturing patient information at the participating practices, the data required extensive and intensive cleaning efforts to extract information and standardize language and terms. For example, check bump, dog has a bump on leg, bumps, lump(s), mass(es), growth(s), and longer verbiage of such descriptions were all simplified to one standard term, mass. Additionally, the SNOMED ${ }^{\circledR}$ (Systemized Nomenclature of Medicine) extension Veterinary Terminology Services Laboratory was used to determine the clinical terminology to apply to the terms for categorization of similar complaints (e.g. lameness vs.

limping). ${ }^{15}$ This ensured the analysis of terminology was scientifically-based in accepted evidence-based 
language which is important for the internal standardization of the linguistic differences in reporting. The data was captured in an Excel spreadsheet and sorted using Pivot tables.

\section{Declarations}

Ethics Approval and Consent to Participate: Institutional Review Board Approval was provided by Lincoln Memorial University Office of Grants and Sponsored Projects (IRB 773 V.0). Informed consent and nondisclosure agreements were obtained and signed between participating sites and Lincoln Memorial University prior to conducting the research.

Consent for Publication: Not Applicable

Availability of Data and Materials: The datasets used and/or analyzed during the current study are available from the corresponding author on reasonable request.

Competing Interests: The authors declare that they have no competing interests.

Funding: This work was supported through financial contributions from The Stanton Foundation.

Acknowledgments: A special thank you to all the veterinary clinics who participated in this study. The authors also acknowledge and appreciate the work of Dr. Karen Gruszynski, CAHA Epidemiologist at Lincoln Memorial University, College of Veterinary Medicine, who provided insight to the study's researchers on data gathering and analysis.

\section{References}

1. Osburn BI, Betts J, Granstrom DE, et al. Roadmap for Veterinary Medical Education in the 21st Century: Responsive, Collaborative, Flexible. NAVMEC North American Veterinary Medical Education Consortium. https://www.aavmc.org/data/files/navmec/navmec_roadmapreport_web_booklet.pdf. Accessed 19 Feb 2020

2. Stull JW, Shelby JA, Bonnett BN, et al. Barriers and next steps to providing a spectrum of effective health care to companion animals. J Am Vet Med Assoc. 2018; doi:10.2460/javma.253.11.1386.

3. Block G. A new look at standard of care. J Am Vet Med Assoc. 2018; doi:10.2460/javma.252.11.1343.

4. Grimm H, Bergadano A, Musk GC, Otto K, Taylor PM, Duncan JC. Drawing the line in clinical treatment of companion animals: recommendations from an ethics working party. BMJ Journals Veterinary Record. 2018; doi:1136/vr.104559.

5. Kipperman BS, Kass PH, Rishniw M. Factors that influence small animal veterinarians' opinions and actions regarding cost of care and effects of economic limitations on patient care and outcome and professional career satisfaction and burnout. J Am Vet Med Assoc. 2017;250:785-794. 
6. AVMA American Veterinary Medical Association. Principles of Veterinary Medical Ethics of the AVMA. 2020. https://www.avma.org/policies/principles-veterinary-medical-ethics-avma. Accessed 19 Feb 2020.

7. Hernandez E, Fawcett A, Brouwer E, Rau J, Turner PV. Speaking Up: Veterinary Ethical Responsibilities and Animal Welfare Issues in Everyday Practice. Animals (Basel). 2018; doi:10.3390/ani8010015

8. O'Neill DG, Church DB, McGreevy PD, Thomson PC, Brodbelt DC. Prevalence of Disorders Recorded in Dogs Attending Primary-Care Veterinary Practices in England. PLoS ONE. 2014; doi:10.1371/journal.pone.0090501.

9. McGreevy PD, Wilson BJ, Mansfield CS, et al. Labrador retrievers under primary veterinary care in the UK: demography, mortality and disorders. BMC Canine Genetics and Epidemiology. 2018; doi:10.1186/s40575-018-0064-x.

10. O'Neill DG, Packer RMA, Lobb M, et al. Demography and commonly recorded clinical conditions of Chihuahuas under primary veterinary care in the UK in 2016. BMC Vet Res. 2020; doi:10.1186/s12917-020-2258-1.

11. Banfield Pet Hospital. State of Pet Health Report. 2019. https://www.banfield.com/state-of-pethealth. Accessed 19 Feb 2020.

12. Top 10 reasons pets visit veterinarians. J Am Vet Med Assoc. 2006. https://www.avma.org/javmanews/2006-09-01/top-10-reasons-pets-visit-veterinarians. Accessed 15 Dec 2019.

13. Most Common Medical Conditions that Prompt Veterinary Visits. Now from Nationwide. 2018. https://blog.nationwide.com/news/reasons-to-take-pets-to-the-vet/. Accessed 15 Dec 2019.

14. North American Pet Health Insurance Association (NAPHIA). State of the Industry Report. 2019. https://naphia.org/downloads/NAPHIA-State-of-the-Industry-2019.pdf. Accessed 19 Feb 2020

15. Veterinary Terminology Services Laboratory, SNOMED. 2019. https://vtsl.vetmed.vt.edu/. Accessed 15 Dec 2019.

\section{Tables}

\begin{tabular}{|l|r|r|r|r|r|}
\hline \multicolumn{7}{|c|}{ Table 1 } \\
Top 10 Complaints by Sex & & \\
\hline Complaint & Female Spayed & \multicolumn{1}{c|}{ Female } & Male Neutered & Male & Unknown \\
\hline Ear & 603 & 109 & $\mathbf{5 4 6}$ & 187 & $\mathbf{2 6}$ \\
\hline Vomiting & 545 & 173 & 426 & 223 & 92 \\
\hline Skin & 549 & 142 & 478 & 219 & 60 \\
\hline Mass & 459 & 92 & 397 & 103 & 71 \\
\hline Diarrhea & 380 & 119 & 328 & 175 & 43 \\
\hline Not Eating & 358 & 101 & 249 & 131 & 115 \\
\hline Eye & 340 & 84 & 327 & 96 & 40 \\
\hline Lameness & 362 & 66 & 303 & 106 & 40 \\
\hline Lethargic & 272 & 71 & 226 & 101 & 93 \\
\hline Coughing & 232 & 56 & 213 & 89 & 43 \\
\hline Total & $\mathbf{4 1 0 0}$ & $\mathbf{1 0 1 3}$ & $\mathbf{3 4 9 3}$ & $\mathbf{1 4 3 0}$ & $\mathbf{6 2 3}$ \\
\hline
\end{tabular}

\section{Figures}




\section{Top Owner-Reported Complaints}

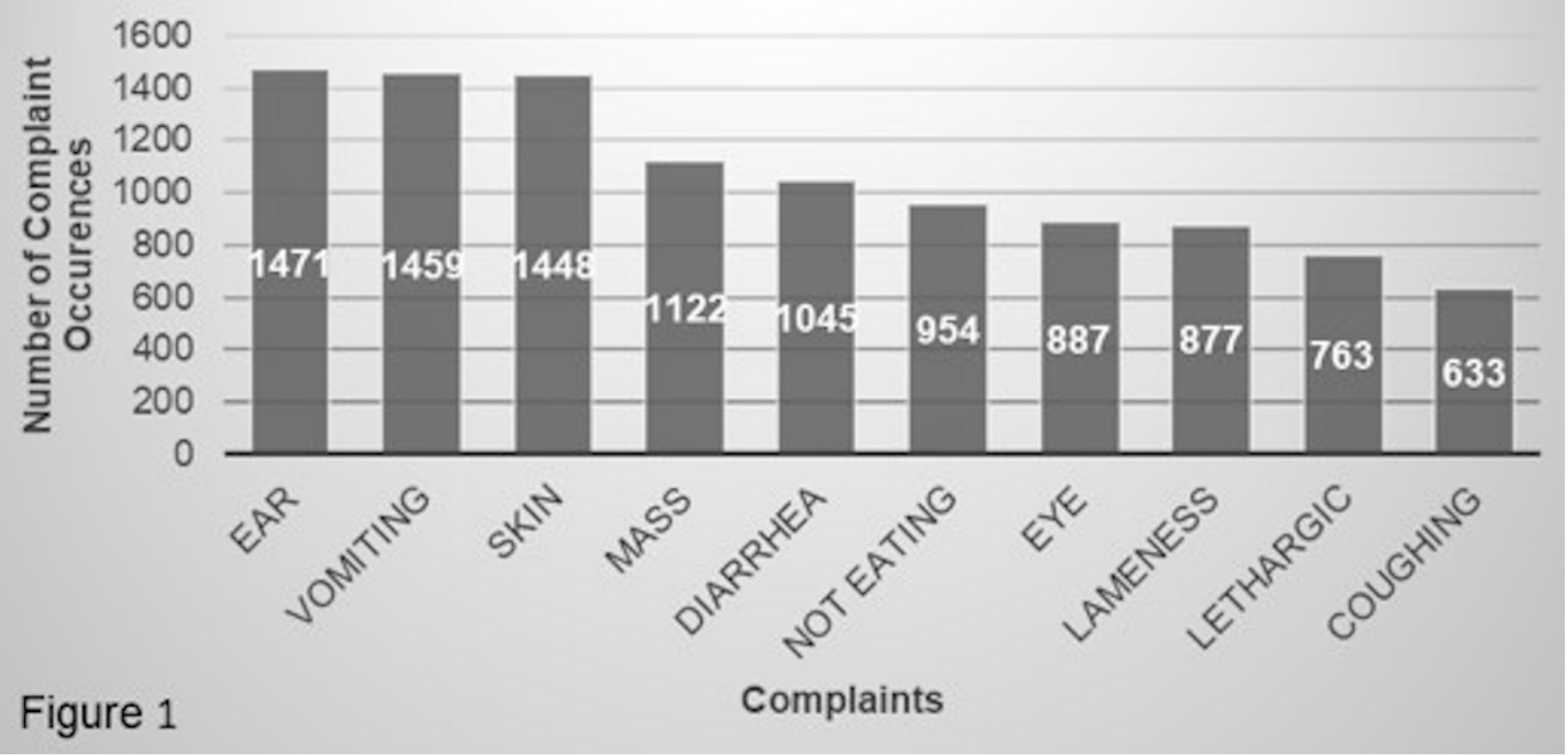

Figure 1 


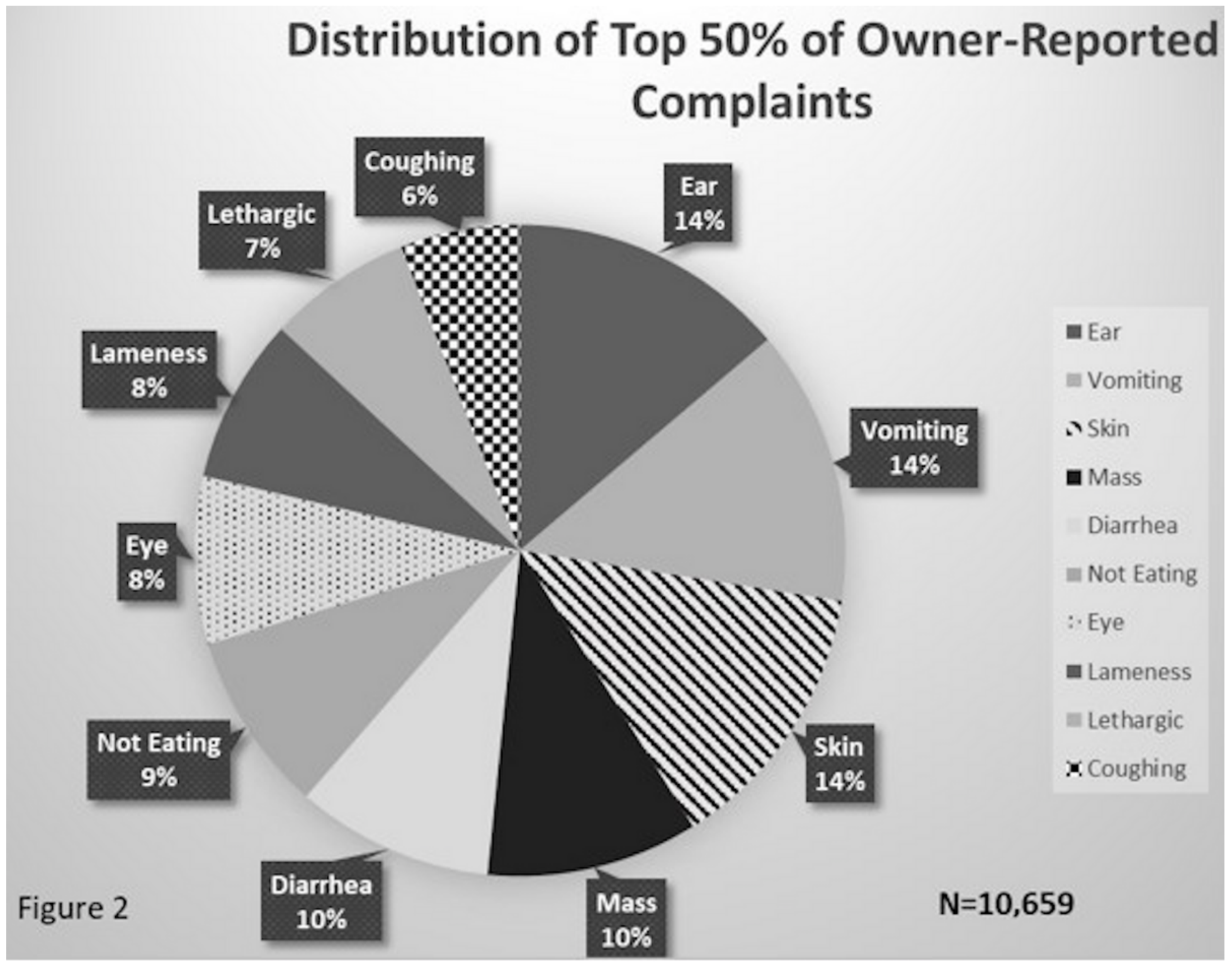

Figure 2 


\section{Study Population by Sex}

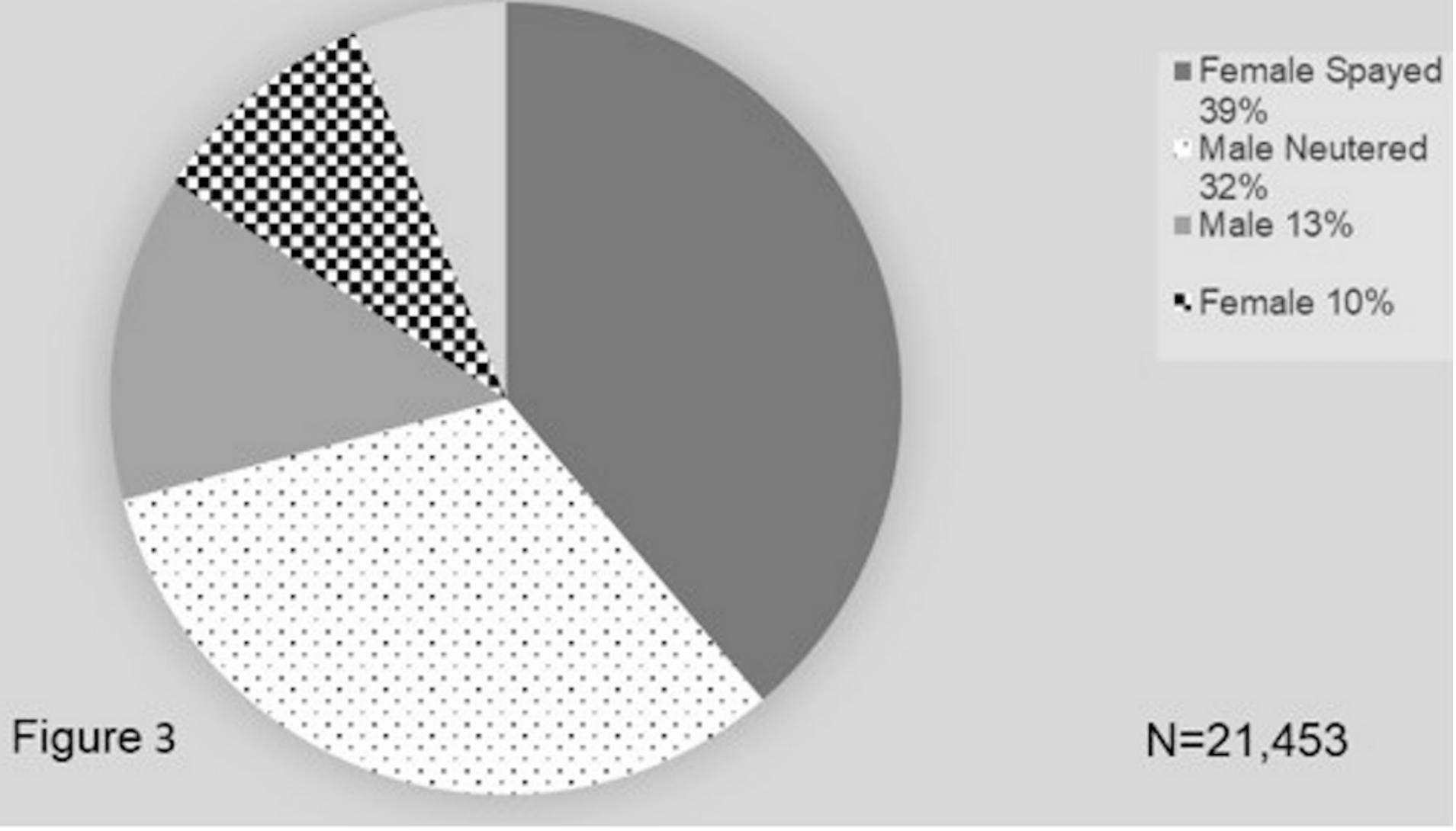

Figure 3 


\section{Top 10 Complaints by Sex}

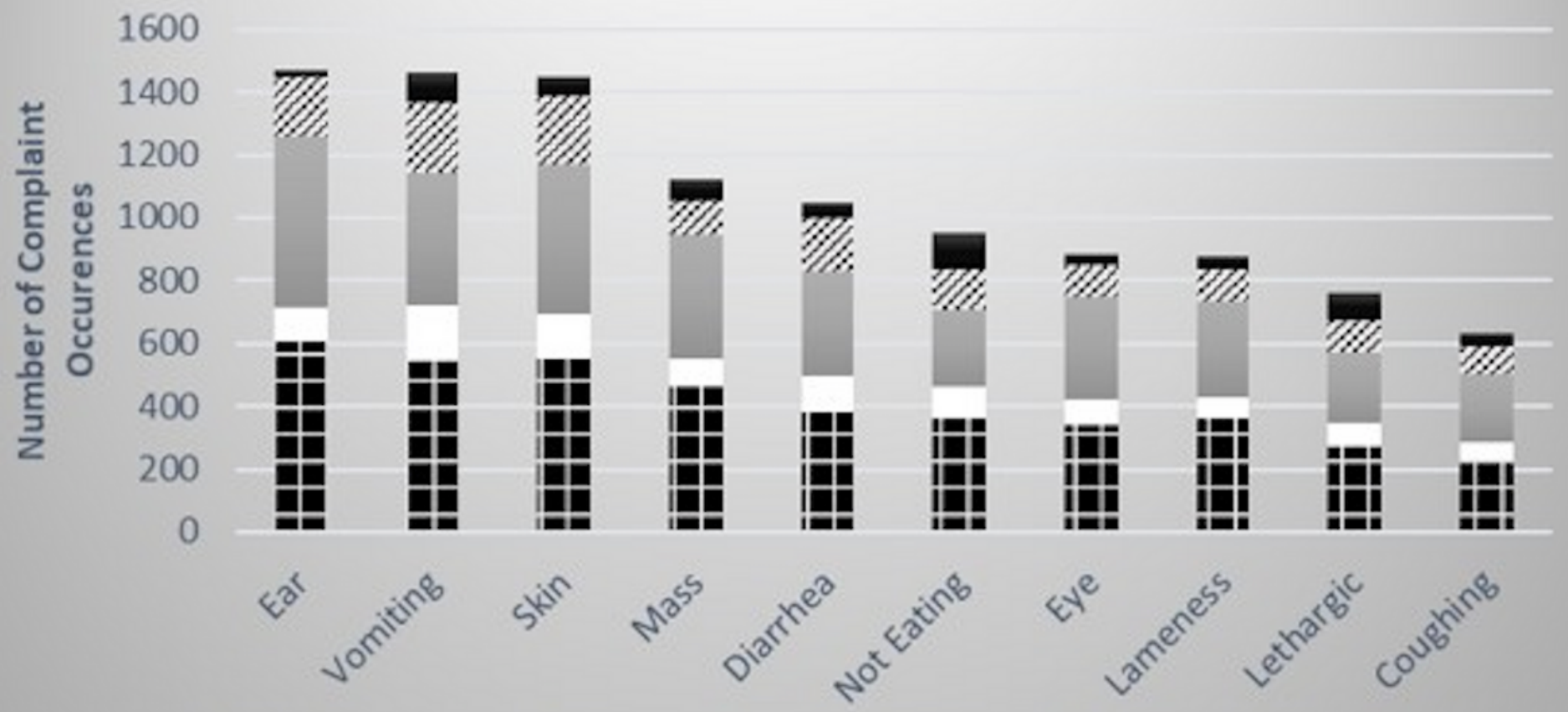

Figure 4

Figure 4 